\title{
A NEW WAY THAT PLANETS CAN AFFECT THE SUN
}

by

Charles Wolff/671 and Paul Patrone/UMD

\begin{abstract}
POPULAR SUMMARY
As planets orbit the Sun, the Sun also has to move to keep the total momentum of the solar system constant. The Sun's small orbital motion plus its 25 day rotation about its axis combine to invigorate some solar instabilities. Occasional convection cells at the proper phase in their short life can be strengthened by factors of two or more. This local burst of extra kinetic energy eventually reaches the surface where it can increase the intensity of solar activity. It might explain some reports in the last century of how planetary positions correlate with solar activity. This is the first effect of planets that is large enough to cause a significant response on the Sun.
\end{abstract}

